\title{
Changing Perspectives in Diabetic Macular Oedema - Recognising and Understanding Chronic Diabetic Macular Oedema
}

\author{
Proceedings of a Symposium Presented at the 14 EURETINA Congress, \\ London, 12 September 2014
}

Expertly Reviewed by: Usha Chakravarthy ${ }^{1}$

Symposium Speakers: Albert Augustin, ${ }^{2}$ Yit Yang, ${ }^{3}$ Michael Diestelhorst ${ }^{4}$ and Pascale Massin ${ }^{5}$

1. Ophthalmology and Vision Sciences, Queen's University Belfast, UK; 2. Augenklinik Karlsruhe, Karlsruhe, Germany; 3. Wolverhampton Eye Infirmary, New Cross Hospital, Wolverhampton, UK; 4. Department of Ophthalmology, University of Cologne, Cologne, Germany; 5. Hôpital Lariboisière, Paris, France

\begin{abstract}
The satellite symposium moderated by Usha Chakravarthy entitled 'Changing Perspectives in Diabetic Macular Oedema: Recognising and Understanding Chronic Diabetic Macular Oedema' was convened at the 2014 EURETINA Congress. The symposium discussed the multiple processes involved in chronic diabetic macular oedema (DMO) and the use of medications, in particular, corticosteroids in its management. As DMO progresses, inflammatory cytokines are up-regulated relative to vascular endothelial growth factor (VEGF) and these promote various pathways that ultimately result in retinal damage in chronic disease. It is important therefore that treatments for chronic DMO address this altered inflammatory cytokine profile to effectively manage the condition. ILUVIEN ${ }^{\circledR}$, a $190 \mu \mathrm{Mg}$ intravitreal implant in applicator (with a daily release rate of $0.2 \mu \mathrm{g} /$ day fluocinolone acetonide [FAc] implant) is a second-line therapy indicated for the treatment of chronic DMO. This implant has been shown in phase III trials to lead to marked improvements in visual acuity and in retinal thickness in patients with chronic DMO that were insufficiently response to first-line therapy (i.e. laser). Clinical trial data strongly support the use of the FAc implant in chronic DMO and now 'real world' data from its use in regular clinical practice are becoming available and interim results complement those reported in a clinical trial setting.
\end{abstract}

\section{Keywords}

Diabetic macular oedema, intravitreal corticosteroid, $190 \mu \mathrm{g}$ fluocinolone acetonide intravitreal implant in applicator (ILUVIEN), visual acuity, FAME studies, FAMOUS studies

\begin{abstract}
Disclosure: Usha Chakravarthy, Albert Augustin, Yit Yang, Michael Diestelhorst and Pascale Massin have attended advisor boards and speaker engagements and have been remunerated for these by Alimera Sciences Ltd.

Acknowledgements: Editorial assistance was provided by James Gilbart at Touch Medical Media, London, UK.

Received: 22 October 2014 Accepted: 18 November 2014 Citation: European Ophthalmic Review, 2014;8(2):145-51 DOI: 10.17925/EOR.2014.08.02.145

Correspondence: Usha Chakravarthy, Clinical Professor, Centre for Experimental Medicine, School of Medicine, Dentistry and Biomedical Sciences, Queen's University,

Northern Ireland, UK. E: u.chakravarthy@qub.ac.uk
\end{abstract}

Support: The publication of this article was funded by Alimera Sciences Ltd. The views and opinions expressed are those of the authors and not necessarily those of Alimera.

Diabetic macular oedema (DMO) is a serious threat to vision that is showing increasing prevalence as a consequence of the worldwide increase in both type I and type II diabetes.' DMO affects approximately $7 \%$ of people with diabetes and is responsible for $12 \%$ of new cases of blindness each year. ${ }^{2,3}$ In many cases, diabetes leads to diabetic retinopathy (DR) approximately 10 years after disease onset and this can lead to DMO. In some cases, however, DMO can occur without DR. Effective means of controlling DMO to prevent widespread vision loss is a growing need. Until recently, the only treatment that was available for the management of DR and DMO was argon laser photocoagulation but now new pharmacological treatments are emerging that are improving the understanding of this disease and enabling better patient outcomes. ${ }^{4,5}$ Two classes of intravitreal injections include inhibitors of vascular endothelial growth factor (VEGF) and corticosteroids. These treatments provide a much improved choice for the management of DMO and, in the case of corticosteroid treatments, can sustain long-term improvements (in visual acuity and reduced macular oedema) for up to 3 years. ${ }^{6}$ In some patients, such medicines are used too late in the disease course and optimal outcomes in terms of vision and retinal thickness are consequently not achieved. ${ }^{7-9}$ This is one of the matters that was discussed at the symposium held by Alimera Sciences at the 2014 EURETINA congress in London. This article reports the proceedings from this symposium.

\section{Objectives of the Symposium}

The symposium aimed to consider the inflammatory processes involved in chronic $\mathrm{DMO}$, to review the clinical trial data supporting the use of fluocinolone acetonide (FAC) implant therapy and to report important interim findings from a 'real world' study of this treatment in early regular clinical use (non-randomised, open label, single-centred, phase IV efficacy and safety study) that is in progress at a treatment centre in France. 


\title{
The Role of Inflammation in Chronic Diabetic Macular Oedema
}

\author{
Albert Augustin, Augenklinik Karlsruhe, Karlsruhe, Germany
}

As diabetes progresses over time inflammatory complications, including DR and DMO, substantially worsen. ${ }^{10}$ In many patients DR appears first and this subsequently leads to DMO. In some cases DMO has been observed without DR suggesting alternative routes to this condition. When DR manifests, increased circulating levels of VEGF are initially observed but as the severity of the condition worsens (decreasing Early Treatment Diabetic Retinopathy Study [ETDRS] scores) these levels remain relatively stable. ${ }^{11,12}$ However, levels of other inflammatory markers, such as procoagulants, markers of endothelial dysfunction and angiogenesis increase relative to VEGF.13,14 As a consequence, the duration of diabetes has been correlated with the development and progression of DR. This was shown in the Wisconsin Epidemiology Study of Diabetic Retinopathy, ${ }^{15}$ which included 1,585 patients with diabetes, who were taking insulin and were diagnosed with DR before 30 years of age. This population-based study showed that progression to proliferative retinopathy and clinically significant macular oedema was significantly related to increasing severity of retinopathy at baseline $(p<0.001) .{ }^{16}$ Further evidence of this was shown in the Diabetes Control and Complications Trial (DCCT correlated) ${ }^{17}$ that included 1,441 patients with insulin-dependent diabetes, type 1 diabetics, aged 13-39 years. The results indicated that during the 8.5 years of follow-up, DR increased with duration of diabetes but the cumulative incidence of DR was significantly decreased with intensive diabetes therapy (i.e. with an external insulin pump or by three or more daily insulin injections) compared with conventional therapy (one or two daily insulin injections).

In patients with diabetes there are multiple processes leading to DR and DMO among which inflammation is important but is not the only mechanism involved. Clinical studies have shown that the effects of diabetes are multifactorial: intraocular levels of various cytokines associated with inflammation and angiogenesis increase with greater severity of DR. The main interleukins that are associated with DR and

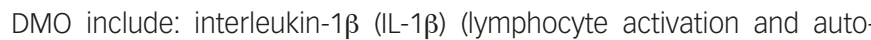
inflammation), IL-6 (increases vascular permeability) and IL-8 (increases vascular permeability and simulates inflammatory proteins). ${ }^{18,19}$ Other important cytokines in DR and DMO are monocyte chemo-attractant protein-1 (MCP-1, increases vascular permeability altering tight junctions increasing cellular recruitment), interferon gamma-induced protein-10 (IP-10, a chemo-attractant for monocytes, macrophages, T cells, natural killer cells and dendritic cells), platelet-derived growth factor (PDGF, promotes embryonic development, cell proliferation, cell migration, and angiogenesis) and VEGF (stimulates angiogenesis, vasodilation and is a chemo-attractant for macrophages and granulocytes)..$^{20-24}$

A study by Dong et al. ${ }^{11}$ showed that among consecutive patients with diabetes $(n=136)$, levels of IL-1 $\beta, I L-6, I L-8, M C P-1$ and IP-10 in the aqueous humor increased with the severity of DR. These levels were also significantly higher in patients with type II diabetes compared with patients without diabetes controls ( $p<0.001$ for all these cytokines). With increasing severity of DR (10-80 on the ETDRS scale) there was little increase in VEGF levels ( $p=0.733)$, but there were a significant increases in levels of IL-1 $\beta(p=0.003), I L-6 \quad(p<0.001), I L-8 \quad(p=0.001)$, MCP-1 $(p<0.001)$ and IP-10 $(p<0.001)$. Levels of many of these cytokine factors can be reduced with intraocular steroid therapy. A

\section{Table 1: The Effect of Intravitreal Steroid (Triamcinolone Acetonide) and Intravitreal Bevacizumab on Cytokine Factors of Inflammation and Angiogenesis}

\begin{tabular}{lllllll}
$\begin{array}{l}\text { Cytokine } \\
\text { conc. } \\
\text { (pg/ml) }\end{array}$ & $\begin{array}{l}\text { Pre- } \\
\text { injection }\end{array}$ & $\begin{array}{l}\text { Post- } \\
\text { injection }\end{array}$ & p Value* & $\begin{array}{l}\text { Pre- } \\
\text { injection }\end{array}$ & $\begin{array}{l}\text { Post- } \\
\text { injection }\end{array}$ & p Value* \\
IL-6 & 29.9 & 13.8 & $<0.01$ & 26.7 & 24.0 & 0.477 \\
\hline IL-8 & 28.2 & 25.3 & 0.597 & 23.9 & 23.6 & 0.374 \\
IP-10 & 366 & 249 & 0.013 & 401 & 433 & 0.110 \\
\hline MCP-1 & 3,850 & 1,090 & 0.010 & 3,770 & 3,840 & 0.594 \\
\hline PDGF-AA & 68.7 & 37.1 & 0.016 & 81.0 & 72.7 & 0.722 \\
\hline VEGF & 55.0 & 10.5 & 0.050 & 61.5 & 0.1 & $<0.01$
\end{tabular}

*Wilcoxon signed rank test. $I L=$ interleukin; $I P=$ interferon-inducible protein; IVBe = intravitreal bevacizumab; IVTA = intravitreal triamcinolone acetonide $M C P=$ monocyte chemotactic protein: $P D G F=$ platelet-derived growth factor: $V E G F=$ vascular endothelial growth factor. Information source: Sohn et al., 2011.12

study by Sohn et al..$^{12}$ showed that in aqueous humor samples, levels of cytokines were significantly raised in patients with DMO. Eleven patients (mean age 54.5 years, with type ॥ diabetes, DR, DMO and no previous vitreous surgery or photocoagulation) were treated with intravitreal triamcinolone acetonide (TA) in one eye and intravitreal bevacizumab in the other eye. Aqueous humor samples taken 4 weeks later showed that in the eyes treated with TA, there were significantly reduced concentrations of IL-6, IL-10, IP-10, MCP-1, PDGF and VEGF. This demonstrated the multifactorial effect of corticosteroid treatment. The eyes treated with bevacizumab, however, showed only a specific reduction in VEGF $(p \leq 0.05)$ but no reduction in the other cytokines monitored (see Table 1).

A main approach to treating DMO is with the use of intravitreal antiVEGF therapy such as ranibizumab ${ }^{25}$ or the recently approved agent, aflibercept. ${ }^{26}$ Ranibizumab is a monoclonal antibody therapy that is approved for DMO and was evaluated in the RIDE (NCT00473382) and RISE (NCT0047330) studies ( $n=759$ ). Patients with DMO were originally randomised to ranibizumab $0.3 \mathrm{mg} /$ day, $0.5 \mathrm{mg} /$ day or sham treatment. ${ }^{27}$ After 24 months, there were marked differences between ranibizumaband sham-treated groups in terms of visual acuity (VA) (improvements in mean best-corrected VA [BCVA] from baseline for ranibizumab 0.5 mg, 0.3 mg and sham/0.5 mg were 12.0, 10.9 and 2.3 for RIDE, and 12.5, 11.9 and 2.6 for RISE, respectively). At 24 months, the sham-treated patients were switched to ranibizumab $0.5 \mathrm{mg}$ /day. Over the following 12 months, however, the patients who were switched failed to show the level of improvements in BCVA scores that were seen in the patients treated with ranibizumab from the start (improvements in BCVA scores from baseline for ranibizumab $0.5 \mathrm{mg}, 0.3 \mathrm{mg}$ and sham/0.5mg were $11.4,10.6$ and 4.6 for RIDE, and 14.2, 11.0 and 4.3 for RISE, respectively). This showed that delaying ranibizumab in DMO may have a negative effect on outcomes and that this treatment may be less effective in more advanced disease. These results are consistent with the principle discussed above that in chronic $\mathrm{DMO}$, inflammation is up-regulated and to achieve a sufficient response a multifactorial approach, targeting multiple inflammatory cytokines, may be needed. 
Further evaluation of ranibizumab was provided by results from the Ranibizumab (intravitreal injections) in RESTORE extension study, (NCT00687804), which in addition to primary visual acuity endpoints, enabled a comparison of early administration of ranibizumab versus 12-month delayed ranibizumab in the treatment of DMO. ${ }^{28}$ Patients were originally randomised to ranibizumab $0.5 \mathrm{mg} /$ day, ranibizumab $0.5 \mathrm{mg}$ / day + laser or laser alone for 12 months. Patients $(n=240)$ then entered the 24-month open-label extension study in which ranibizumab-treated patients continued treatment but laser-treated patients were switched to ranibizumab. At the end of the 12-month period, the mean change in BCVA from baseline in the three groups were 7.9, 7.1 and 2.3, respectively. At the end of the extension study (at 24 months), however, these scores were: 8.0, 6.7 and 6.0. This showed that patients who crossed over from laser to ranibizumab treatment at 12 months experienced the same benefit as those who were treated early. It should be noted, however, that patients recruited to this study were not required to have persistent DMO so the cohort included those with early-stage disease. In this study, therefore, antiVEGF therapy may have been effective in early-stage DMO but for more progressive disease, this switching strategy was not effective. Therefore, in more chronic cases, the response to anti-VEGF may be blunted and a second-line therapy such as a corticosteroid should be considered.

The above responses have been observed in the Fluocinolone Acetonide for Diabetic Macular Edema (FAME) studies. ${ }^{29}$ Indeed, in the sham control group, rescue laser (61.6 and $62.5 \%$ ) and off-protocol treatments (34.8 and $30.6 \%$ ) were given in similar proportions to chronic and non-chronic patients, respectively. However, improvements were quite different with only $13.4 \%$ of chronic DMO patients achieving $\geq 15$-letter in BCVA from baseline compared with $27.8 \%$ of non-chronic DMO patients. Thus, intermittent therapies, such as rescue laser and off-protocol treatments, were effective in patients in whom DMO had been diagnosed more recently but less effective in patients with longer standing DMO. This indicates that different treatment approaches are needed to successfully manage chronic DMO and achieve optimal outcomes for the patient.

Albert Augustin: "AS DR worsens with time, the incidence of DMO increases which, in chronic cases, is no longer mediated by VEGF but by pro-inflammatory cytokines. In these patients, steroids provide an effective therapeutic option"

\section{Take-home Messages}

- $\quad$ Time is the greatest risk factor for worsening DMO

- As DR worsens, DMO increases (but in some cases, DMO can occur in the absence of DR)

- $\quad$ Relative to VEGF, multiple inflammatory cytokines are upregulated as DR worsens

- In some patients, especially chronic disease, DMO is no longer mediated by VEGF alone

- $\quad \mathrm{DMO}$ that is mediated by multiple cytokines can be treated effectively with intravitreal steroids, as it is multifactorial

\section{Overview of ILUVIEN ${ }^{\circledR}(0.2 \mu \mathrm{g} /$ Day Fluocinolone Acetonide Implant $)$ in Chronic Diabetic Macular Oedema}

Yit Yang, Wolverhampton Eye Infirmary, New Cross Hospital, Wolverhampton, UK

ILUVIEN® (FAC implant) is a very long-acting, second-line therapy indicated for the treatment of chronic DMO. The product consists of a non-bioerodable micro implant (dimensions: $3.5 \mathrm{~mm} \times 0.37 \mathrm{~mm}$ ) that contains $190 \mu \mathrm{g}$ of FAc and delivers $0.2 \mu \mathrm{g}$ FAc/day for up to 36 months. The implant is administered intravitreally using a 25-gauge injector, which creates a self-sealing wound. After a brief burst, levels of FAc in the aqueous humor have been shown to stabilise by 6 months $(1.18 \mathrm{ng} / \mathrm{ml})$ and are sustained through to 36 months $(0.55 \mathrm{ng} / \mathrm{ml}){ }^{30,31}$ The FAME studies recruited patients with DMO that was unresponsive to previous macular laser treatment. Patients were also required to have a foveal thickness $\geq 250 \mu \mathrm{m}$ despite at least one prior focal/grid macular laser photocoagulation treatment and BCVA in ETDRS letter score between 19 and 68 (Snellen equivalent range, 20/50-20/400). ${ }^{32}$

Figure 1: The Effect of Fluocinolone Acetonide Implant versus Control Treatment on the Proportion of Patients with $\geq 15$-letter Improvement in the FAME Study in Individuals from A. Whole Study Population and B. Chronic Diabetic Macular Oedema Subgroup

A

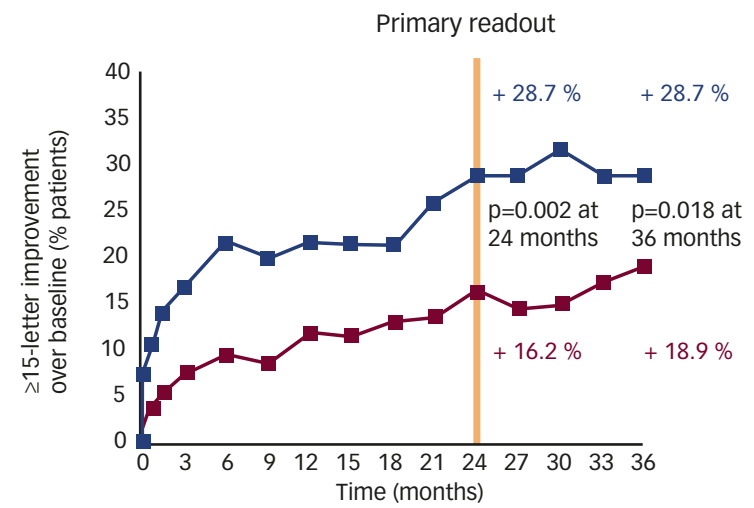

B

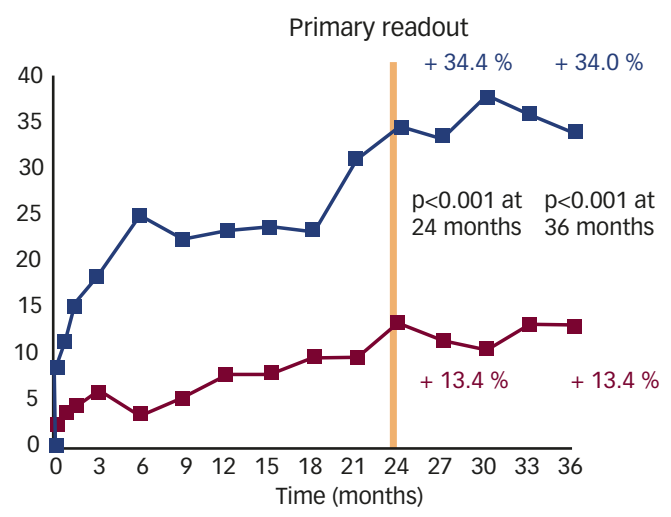

FAME = Fluocinolone Acetonide for Diabetic Macular Edema. Adapted from Cunha-Vaz et al., 2014;:29 and Campochiaro et al., $2012 .{ }^{30}$ 
Treatment with $0.2 \mu \mathrm{g} /$ day FAc implant $(n=375)$ led to rapid improvements in retinal thickness compared with sham (control) treatment $(n=185)$. At 6 weeks, foveal thickness had rapidly decreased from 461.1 to $345.7 \mu \mathrm{m}$ $(-115.4 \mu \mathrm{m})$ in the $0.2 \mu \mathrm{g} /$ day FAc implant-treated group compared with a change from $451.3 \mu \mathrm{m}$ to $450.4 \mu \mathrm{m}(-0.9 \mu \mathrm{m})$ in the sham control group. VA was also significantly improved by the FAc implant. The proportions of patients with a $\geq 15$-letter improvement after 24 months was $28.7 \%$ and $16.2 \%(\Delta, 12.5 \% ; p=0.002)$ and after 36 months was $28.7 \%$ and $18.9(\Delta, 9.8 \% ; p=0.018)$ in FAc implant- and sham-treated patients, respectively. ${ }^{30}$ The FAME studies also show the efficacy of the FAc implant is greatest in patients with chronic DMO. This is the population in which the FAc implant is indicated. In these patients the proportions with a $\geq 15$-letter improvement at 36 months were $34.0 \%$ and $13.4 \%$, respectively $(\Delta, 20.6 \% ; p<0.001)$ (see Figure 1). ${ }^{29,30}$ These results indicate that the FAc implant is an effective treatment in a group of patients who have not responded to prior treatment with first-line therapies such as laser.

Common side effects of corticosteroids are the potential for cataract and raised intraocular pressure (IOP). These were both managed effectively in the FAME studies. ${ }^{33}$ Indeed, $38.4 \%$ of FAc implant-treated patients needed IOP-lowering drops compared with $14.1 \%$ of controls. Therefore $62 \%$ of patients treated with $0.2 \mu \mathrm{g} /$ day did not require IOP therapy and when IOP-lowering treatment was given, it did not affect vision. This was demonstrated in the FAME studies, where the mean change in BCVA was similar when patients receiving chronic IOPlowering therapy (taken for $>28$ days; $n=144$ ) were compared with those that were not $(n=230){ }^{34}$

It should be noted that the UK National Institute for Health and Clinical Excellence (NICE) recommends the FAc implant for use in pseudophakic patients due to the associated cost of performing cataract surgery. ${ }^{35,36} \mathrm{In}$ other countries in Europe, such as Germany, there is no restriction on lens status and the FAc implant can be used as indicated in the ILUVIEN Summary of Product Characteristics.

Yit Yang: "IOP rise during steroid treatment is not a disaster - this and cataract removal can be managed with good visual outcomes"

\section{Take-home Messages}

- The FAc implant is a very long-acting treatment for DMO

- The FAc implant rapidly resolves oedema and improves VA

- Efficacy is greatest in patients with chronic DMO

- $\quad$ Secondary IOP rise is managed with good visual outcomes

\section{Understanding and Managing Steroid-induced Elevation of Intraocular Pressure in the Context of the FAME Studies}

Michael Diestelhorst, University of Cologne, Cologne, Germany

One side effect of chronic topical or systemic treatment with steroids is reduced outflow facility of the trabecular meshwork. As a consequence, the IOP may rise significantly within weeks. If not treated appropriately these eyes may show significant glaucomatous optic nerve head (ONH) cupping. Thus steroid glaucoma could be termed 'high-speed open angle glaucoma'. It may cause blindness within months. Such patients have no pain even when the IOP is $50 \mathrm{mmHg}$. When possible, the steroid application should be stopped. If the $\mathrm{ONH}$ and visual field (VF) are normal and the IOP is up to $25 \mathrm{mmHg}$, eyes should be controlled without antiglaucomatous medication. At higher IOP levels, topical or systemic IOP-lowering therapy is needed. If this does not reduce the IOP level to normal, ocular surgery should be considered.

Elevated IOP is often associated with intravitreal corticosteroid treatment. In the FAME studies, steroid-induced ocular hypertension was limited and controlled among patients who received an FAc implant. Among the FAC implant-treated eyes, $43.1 \%$ had a maximum IOP $<21 \mathrm{mmHg}$ (it should be noted that patients with IOP $>21 \mathrm{mmHg}$ or those receiving IOP-lowering drops at baseline were excluded). ${ }^{34}$ Patients receiving this treatment with an IOP up to $25 \mathrm{mmHg}$ are considered to be 'low responder' and could be compared with 'ocular hypertensives' when the VF and the $\mathrm{ONH}$ are normal (see European Glaucoma Society Guidelines). ${ }^{37}$ In the FAME studies, $67.3 \%$ of patients had an IOP $<25 \mathrm{mmHg}$. The proportion of patients with an IOP above $30 \mathrm{mmHg}$ ('high-responder') in the FActreated group was $18.4 \%$ versus $4.3 \%$ in the sham-control group.

Analysis of the time to the first treatment with IOP-lowering drops shows that roughly $25 \%$ occurred within the first 3 months and $70 \%$

\section{Table 2: Frequencies of Medications Administered for Intraocular Pressure Management During the FAME Studies were Similar to Those Given in Current Clinical Practice}

\begin{tabular}{|c|c|c|}
\hline $\begin{array}{l}\text { Intraocular Pressure-lowering } \\
\text { Medication }\end{array}$ & $\begin{array}{l}\text { Control } \\
(n=185) \%\end{array}$ & $\begin{array}{l}0.2 \mu g / D a y ~ F A c \\
\text { (Licensed Dose) } \\
(n=375) \%\end{array}$ \\
\hline Prostaglandin & 4.9 & 17.3 \\
\hline Beta blocker & 7.0 & 17.6 \\
\hline Topical alpha agonist & 5.4 & 17.9 \\
\hline Topical carbonic anhydrase inhibitor & 2.2 & 12.3 \\
\hline $\begin{array}{l}\text { Topical carbonic anhydrase inhibitor } \\
\text { + beta blocker }\end{array}$ & 4.3 & 11.7 \\
\hline Topical alpha agonist + beta blocker & 1.6 & 8.5 \\
\hline
\end{tabular}

FAC = fluocinolone acetonide implant $;$ FAME = Fluocinolone Acetonide for Diabetic Macular Edema. Source: data on file, Alimera Sciences.

within the first 12 months. ${ }^{30,34}$ However, frequent IOP readings are mandatory within the first 6-12 weeks after treatment is started.

If the IOP increases $>25 \mathrm{mmHg}$, treatment should be initiated. Among FAC implant-treated patients, $62 \%$ did not require IOP-lowering eye drops. A summary of the topical anti-glaucomatous medication prescribed in the FAME studies is shown in Table 2. The most frequent of these were prostaglandins, beta blockers and topical alpha 2 agonists. Combination therapies were also prescribed. The usage of IOP-lowering therapies 
Figure 2: Proportion of Patients with 20/40 Vision or Better or $\geq 15$-letter Improvement in Vision in Patients Treated with $0.2 \mu \mathrm{g} / \mathrm{Day}$ Fluocinolone Acetonide Implant and Grouped by how IOP was Managed

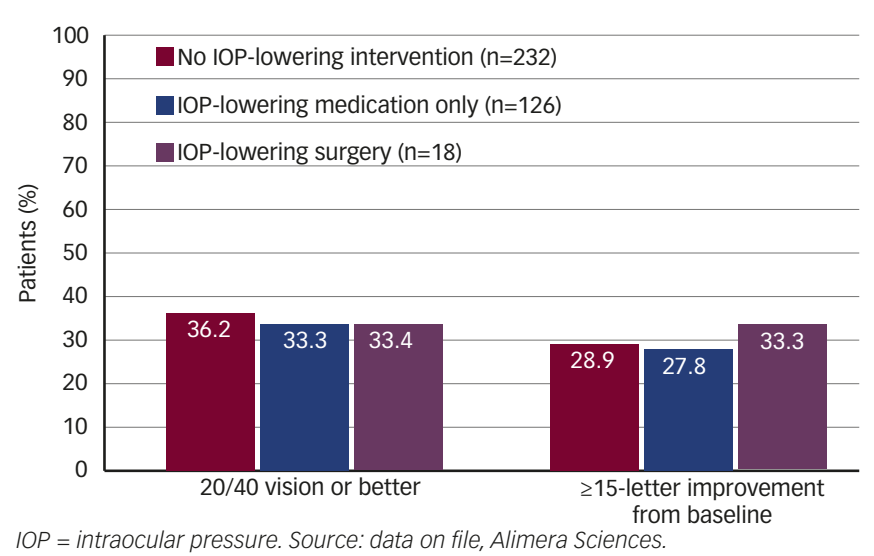

indicates that steroid-induced IOP elevation occurs in a minority of patients who receive the FAc implant. Appropriate monitoring and treatment is important to manage this side effect without detrimental effect on the $\mathrm{ONH}$

In the FAME studies, $95 \%$ of patients treated with an FAc implant did not require incisional IOP-lowering surgery..$^{30}$ Trabeculoplasty was performed in $1.3 \%$ of FAc implant-treated and in $0 \%$ of control patients. Four patients had laser trabeculoplasty 574-776 days after the study start, which successfully reduced IOP. Prior to trabeculoplasty, the IOP was $32.8 \mathrm{mmHg}$ and decreased to $17.5 \mathrm{mmHg}$ after the procedure and was $18.5 \mathrm{mmHg}$ after 360 days. This emphasises that topical anti-glaucomatous therapy is not sufficient in 'high-responders'. Of the four laser patients, three required no incisional treatment. One patient who had been treated with an FAc implant required additional IOP-lowering surgery.

Elevated IOP had little effect on VA. The proportions of patients with $20 / 40$ vision or better or had a $\geq 15$-letter improvement in BCVA score from baseline were similar for those who received IOP-lowering drops or surgery to those who received no IOP-lowering treatment (see Figure 2).

Michael Diestelhorst: "In case of raised IOP: after removal of the FAc implant there is a good chance that IOP will return to normal within weeks"

\section{Take-home Messages}

In the FAME studies:

- $\quad$ Frequency of at least one IOP reading $>30 \mathrm{mmHg}$ in patients who received an FAc implant: $18.4 \%$

- $\quad$ Of the $38 \%$ of patients who received a single FAc implant and required IOP-lowering drops, $70 \%$ did so in the first year

- $\quad 38 \%$ received IOP-lowering drugs at any point; $1.3 \%$ received laser trabeculoplasty for elevated IOP; $4.8 \%$ received incisional surgery to address elevated IOP

- $\quad$ Raised IOP did not adversely affect VA outcomes

\section{Real-world Experience with the $0.2 \mu g / D a y$ Fluocinolone Acetonide Implant}

Pascale Massin, Hôpital Lariboisière, Paris, France

At the time of writing, the FAc implant has been launched in the UK and Germany and licensed in the UK, Germany, Austria, France, Portugal, Spain, Italy, Norway, Sweden and Denmark..$^{38}$ Licences are still pending in the Netherlands, Belgium, Luxembourg, Finland, Ireland, Poland and Czech Republic. ${ }^{39}$ Furthermore, as of 26 September 2014, the FAc implant has been approved for use in DMO by the US Food and Drug Administration (FDA). Current experience of regular clinical use of the FAc implant is limited and this study was conducted to gain real usage experience in France. This study was designed to assess the efficacy and safety of the FAc implant in DMO patients considered insufficiently responsive to available therapies (laser, anti-VEGF) with or without prior history of corticosteroid therapy. Intermediate results after month 1 are reported here.

This phase IV study is an ongoing non-randomised, open-label, singlecentre, investigation in a planned population of 20 eyes. It started in January 2014 and is a 1-year study with assessments at 1 week, and 1, $3,6,9$ and 12 months. At entry, patients were divided into two groups: Group 1, patients with prior laser treatment only (i.e., no anti-VEGF); and, Group 2, patients with prior laser and three or more monthly anti-VEGF treatments. Inclusion criteria included: VA between 20/60 and 20/400, central foveal thickness $\geq 350 \mu \mathrm{m}$ and DMO that was unresponsive to laser photocoagulation ( $\geq 3$ months prior to screening) or unresponsive to anti-VEGF therapy. Exclusion criteria included: IOP $\geq 21 \mathrm{mmHg}$ at screening, a history of IOP increasing to $\geq 25 \mathrm{mmHg}$ following prior intravitreal steroid therapy or the use of $\geq 2$ IOP-lowering drops.

At baseline, patient characteristics in the two groups were similar; however, Group $1(n=7)$ had a longer duration of DMO than Group 2 $(n=10)$ (7.6 versus 3.6 years, respectively) and more than $80 \%$ of patients were pseudophakic. Interim analysis showed a greater gain of VA in Group 2 compared with Group 1, but the duration of chronic DMO was shorter in Group 2 than in Group 1. In terms of anatomical response, decreases in macular thickness were observed in all the eyes in Group 1 as compared with Group 2 where responses were heterogeneous. In Group 2, the average decrease of the macular thickness was less marked versus Group 1. After 1 month, these changes in foveal thickness and BCVA were similar to those seen in the FAME studies.

\section{Take-home Messages}

- In the 'real world' study, the FAc implant was well tolerated in all patients

- $\quad$ Rapid reductions in mean central retinal thickness were seen in both treatment groups following FAc implant treatment

- $\quad$ Improvement in mean VA was observed at month 1 in patients who had received prior treatment with laser and anti-VEGF but not those with prior laser only 


\section{Diabetic Macular Oedema}

To date, the FAc implant has been well tolerated. In all patients, the change in IOP was $\leq 21 \mathrm{mmHg}$. In one patient in Group 1 IOP increased from $19 \mathrm{mmHg}$ at baseline to $30 \mathrm{mmHg}$ by month 1 and this was suspected to be related to the treatment.

Overall, these findings show that the FAc implant was beneficial to patients in this 'real world' study whether or not they had received prior anti-VEGF therapy and laser photocoagulation. The better VA outcomes in Group 2 suggest that it may be beneficial to administer the FAc implant earlier in the disease course. Interim analyses at subsequent time points and at the end of the study will be reported at a future date.

Pascale Massin: "In the two [FAc-treated] groups we observed a reduction in macular thickening." "We now know that we should probably have treated these chronic DMO patients [with FAC] at an earlier stage than we did in this 'real world' study"

\section{Conclusion}

The symposium objectives, see above, were met and valuable insights into chronic DMO pathophysiology and effective treatment with corticosteroids were provided. The FAC implant produces rapid and marked improvements in VA and retinal thickness. The interim 'real world' study results seem to support those found in the FAME studies, which showed marked gains in both VA and retinal pathology in patients with chronic DMO after only 1 month. In particular, these 'real world' data emphasise that the FAc implant is effective after prior treatment with laser and anti-VEGF.

Clinical evidence indicates that several therapies provide benefits in the treatment of DMO but these treatments may be appropriate at different stages of the disease. As DMO progresses and becomes more chronic, the pathophysiology of the disease changes and different inflammatory and angiogenic pathways become activated. While some patients respond well to anti-VEGF therapies, in chronic disease they may become insufficiently responsive meaning a suboptimal response is being achieved. The interim study defined this as being an insufficient response to three or more monthly anti-VEGF treatments. In the interests of the patient, it is appropriate that this insufficient response is detected and a second-line therapy considered. The FAc implant is a second-line therapy and has been shown, in the FAME studies and now in a real-life setting, to produce rapid and marked improvements in vision and foveal thickness in chronic DMO patients that are not responding optimally to prior therapies.

In Europe the FAc implant is approved for chronic DMO patients who are insufficiently responsive to available therapies, while NICE recommends that it should only be used in pseudophakic patients based on costeffectiveness grounds rather than benefit-to-risk.
The administration of intravitreal corticosteroids is associated with cataract, elevated IOP and floaters. While these effects can be adequately managed, the more advantageous safety profiles of the anti-VEGF medications make them more suitable for first-line use. Nevertheless, the administration of FAc implants is an effective therapy for patients who do not respond to initial anti-VEGF treatment.

The FAc implant is a second-line therapy. Experience suggests that it is effective in chronic DMO patients but optimal effects may be gained if the treatment is initiated once a patient has been identified as being insufficiently responsive to prior therapies such as laser and anti-VEGF. Such treatment could dramatically improve outcomes such as gaining 20/40 vision, which is the minimum UK eyesight standard for driving. It could also allow individuals such as bus and lorry drivers to remain in employment (needing VA of at least 0.8 [6/7.5] Snellen scale in the best eye and at least $0.1[6 / 60]$ on the in the other eye ${ }^{40}$.

DMO is an increasingly serious threat to vision worldwide. One FAc implant provides beneficial improvements in VA and foveal thickness that last for up to 36 months with one injection. FAc implants are indicated for use in chronic DMO patients. DMO is a complex disease involving multiple pathways and the FAc implant addresses the multifactorial nature of DMO as it affects multiple inflammatory cytokines that are upregulated as DMO progresses. Early identification of non-responsiveness to prior therapies is particularly helpful for the physician as it helps guide subsequent and appropriate treatment options that meet their patient's needs. It also potentially provides insight into the underlying state of the condition. This helps improve the patient outcomes and potentially helps preserve longer-term vision.
1. Ding J, Wong TY, Current epidemiology of diabetic retinopathy and diabetic macular edema, Curr Diab Rep, 2012;12:346-54.

Klein R, Moss SE, Klein BE, et al., The Wisconsin epidemiologic study of diabetic retinopathy. XI. The incidence of macular edema, Ophthalmology, 1989;96:1501-10.

3. Yau JW, Rogers SL, Kawasaki R, et al., Global prevalence and major risk factors of diabetic retinopathy, Diabetes Care, 2012;35:556-64

4. Kire CA, Porta M, Chong V, Medical management for the prevention and treatment of diabetic macular edema, surv Ophthalmol, 2013;58:459-65.

5. Williams MA, Chakravarthy U, Evidence underlying the clinical management of diabetic macular oedema, Clin Med, 2013;13:353-7.

6. Schwartz SG, Flynn HW, Jr, Scott IU, Emerging drugs for diabetic macular edema, Expert Opin Emerg Drugs, 2014;19:397-405.

7. Bandello F, Casalino G, Loewenstein A, et al., Pharmacological approach to diabetic macular edema, Ophthalmic Res, 2014;51:88-95.

8. Grover D, Li TJ, Chong CC, Intravitreal steroids for macular edema in diabetes, Cochrane Database Syst Rev, 2008;CD005656.

9. Hooper P, Boucher MC, Colleaux K, et al., Contemporary management of diabetic retinopathy in Canada: from guidelines to algorithm guidance, Ophthalmologica, 2014;231:2-15.
10. Girach A, Lund-Andersen H, Diabetic macular oedema: a clinical overview, Int J Clin Pract, 2007;61:88-97.

11. Dong N, Xu B, Wang B, et al., Study of 27 aqueous humor cytokines in patients with type 2 diabetes with or without retinopathy, Mol Vis, 2013;19:1734-46.

12. Sohn HJ, Han DH, Kim IT, et al., Changes in aqueous concentrations of various cytokines after intravitreal triamcinolone versus bevacizumab for diabetic macular edema, Am J Ophthalmol, 2011:152:686-94.

13. Augustin $A$, Loewenstein $A$, Kuppermann $B D$, Macular edema. General pathophysiology, Dev Ophthalmol, 2010;47:10-26.

4. Goldberg RB, Cytokine and cytokine-like inflammation markers, endothelial dysfunction, and imbalanced coagulation in development of diabetes and its complications, J Clin Endocrinol Metab, 2009;94:3171-82.

15. Klein R, Klein BE, Moss SE, et al., The Wisconsin Epidemiologic Study of Diabetic Retinopathy. IX. Four-year incidence and progression of diabetic retinopathy when age at diagnosis is less than 30 years, Arch Ophthalmol, 1989;107:237-43.

16. Henricsson $M$, Sellman $A$, Tyrberg $M$, et al.. Progression to proliferative retinopathy and macular oedema requiring treatment. Assessment of the alternative classification of the Wisconsin Study, Acta Ophthalmol Scand, 1999;77:218-23.

17. The Diabetes Control and Complications Trial Research Group, The effect of intensive treatment of diabetes on the development and progression of long-term complications in insulin-dependent diabetes mellitus, N Engl J Med,
1993;329:977-86.

18. Noma $\mathrm{H}$, Funatsu $\mathrm{H}$, Harino $\mathrm{S}$, et al., Vitreous inflammatory factors in macular edema with central retinal vein occlusion, Jpn J Ophthalmol, 2011:55:248-55.

19. Ramachandran RS, Treatment of retinal vein occlusion. In: FG Holz and RF Spade (eds), Essentials in Ophthalmology London: Springer, 2007;147-63.

20. Funatsu $\mathrm{H}$, Noma $\mathrm{H}$, Mimura T, et al., Association of vitreous inflammatory factors with diabetic macular edema, Ophthalmology, 2009;116:73-9.

21. Lee WJ, Kang MH, Seong M, et al., Comparison of aqueous concentrations of angiogenic and inflammatory cytokines in diabetic macular oedema and macular oedema due to branch retinal vein occlusion, Br J Ophthalmol, 2012;96:1426-30.

22. Meleth $A D$, Agron $E$, Chan $C C$, et al., Serum inflammatory markers in diabetic retinopathy, Invest Ophthalmol Vis SCi, 2005;46:4295-301.

23. Owen LA, Hartnett ME, Soluble mediators of diabetic macular edema: the diagnostic role of aqueous VEGF and cytokine levels in diabetic macular edema, Curr Diab Rep, 2013:13:476-80

24. Praidou A, Papakonstantinou E, Androudi S, et al., Vitreous and serum levels of vascular endothelial growth factor and serum levels of vascular endothelial growth factor in patients with non-proliferative diabetic retinopathy and clinically significant macula oedema, Acta Ophthalmol, clinically significant 
25. Krispel C, Rodrigues $M$, Xin X, et al., Ranibizumab in diabetic macular edema, World I Diabetes, 2013;4:310-8.

26. Moradi A, Sepah YJ, Sadiq MA, et al., Vascular endothelial growth factor trap-eye (Aflibercept) for the management of diabetic macular edema, World I Diabetes, 2013;4:303-9.

27. Brown DM, Nguyen QD, Marcus DM, et al., Long-term outcomes of ranibizumab therapy for diabetic macular edema: the 36-month results from two phase III trials: RISE and RIDE, Ophthalmology, 2013:120:2013-22.

28. Schmidt-Erfurth U, Lang GE, Holz FG, et al., Three-year Schmidt-Erfurth $\mathrm{U}$, Lang $\mathrm{GE}, \mathrm{Holz} \mathrm{FG}$, et al.,
outcomes of individualized ranibizumab treatment in patients outcomes of individualized ranibizumab treatment in patients
with diabetic macular edema: the RESTORE extension study, Ophthalmology, 2014;121:1045-53.

29. Cunha-Vaz J, Ashton P, lezzi R, et al., Sustained delivery fluocinolone acetonide vitreous implants: long-term benefit in patients with chronic diabetic macular edema, Ophthalmology, 2014;121:1892-903.

30. Campochiaro PA, Brown DM, Pearson A, et al., Sustained delivery fluocinolone acetonide vitreous inserts provide benefit for at least 3 years in patients with diabetic macular edema, Ophthalmology, 2012;119:2125-32.

31. Campochiaro PA, Nguyen QD, Hafiz G, et al., Aqueous levels of fluocinolone acetonide after administration of fluocinolone acetonide inserts or fluocinolone acetonide implants, Ophthalmology, 2013;120:583-7.

32. Campochiaro PA, Brown DM, Pearson A, et al., Long-term benefit of sustained-delivery fluocinolone acetonide vitreous inserts for diabetic macular edema, Ophthalmology, 2011;118:626-35 e2.

33. Goldstein DA, Godfrey DG, Hall A, et al., Intraocular pressure in patients with uveitis treated with fluocinolone acetonide in patients with uveitis treated with fluocinolon
implants, Arch Ophthalmol, 2007:125:1478-85.

34. Alimera Sciences, Data on file (accessed 1 September 2014).

35. UK National Institute for Health and Clinical Excellence 2012; Health Technology Appraisal: Fluocinolone acetonide intravitreal implant for the treatment of chronic diabetic macular oedema after an inadequate response to prior therapy. Available at: http://www.nice.org.uk/guidance/ta271/ resources/diabetic-macular-oedema-fluocinolone-acetonideintravitreal-implant-response-to-consultee-commentator-and- public-comments-on-the-appraisal-consultation-documentacd2 (accessed 26 September 2014)

36. Haritoglou C, Neubauer AS, Kernt M, Fluocinolone acetonide and its potential in the treatment of chronic diabetic macular edema, Clin Ophthalmol, 2013;7:503-9.

37. European Glaucoma Society, 2013; Terminology and

Guidelines for Glaucoma 4th edition. Available at: http://www. eugs.org/eng/EGS_guidelines4.asp (accessed 28 September 2014).

38. Pharma Letter, 2012; Alimera's Iluvien approved in France for diabetic macular edema. Available at: http://wmw. for diabetic macular edema. Available at: http://WWw. thepharmaletter.com/article/alimera-s-iluvien-approved-in-
france-for-diabetic-macular-edema. (accessed 4 October 2014)

39. Alimera Sciences, 2014; Iluvien ${ }^{\circledR}$ for Diabetic Macular Edema (DME). Available at: http://www.alimerasciences.com/ products/iluvien-for-diabetic-macular-edema-dme/ (accessed 24 November 2014).

40. UK Government, 2014; Driving eyesight rules, Available at: https://www.gov.uk/driving-eyesight-rules date (accessed 7 October 2104). 